\title{
Erschließung des Conrad-Geßner-Materials der Zentralbibliothek Zürich
}

Durch die Freundlichkeit des Direktors der Zentralbibliothek, Herrn Dr. phil. P.Scherrer, erhielt ich die Erlaubnis, den von Herrn Dr. phil. Rudolf Steiger erstatteten Jahresbericht 1967 über die von ihm ausgeführte Erschließungsarbeit des Conrad-Geßner-Materials der Zentralbibliothek im Gesnerus zum Abdruck zu bringen.

Wer mit der Conrad-Geßner-Forschung in Zürich in den letzten Jahren zu tun hatte, dem wird unvergessen bleiben, welche große Hilfe die durch R.Steiger zu einem eigentlichen Promptuarium erweiterten Nachweise zu Conrad Geßners Leben und Werk gewesen sind. Ein Zettelregister über die im folgenden von Dr. Steiger aufgeführten Gebiete, das noch fortlaufend erweitert wird, hat Dr. Steiger in jahrelanger Bibliotheksarbeit zu einem Promptuarium erweitert, das alles in der Zentralbibliothek Zürich befindliche, der Geßner-Forschung dienende Material nachweist. Außerdem werden darin in der Literatur getroffene Stellen, die für die Erkenntnis von Geßners Leben und Werk von Bedeutung sind, registriert.

Nur wer Dr. Rudolf Steiger an der Arbeit gesehen hat, kann ermessen, mit welcher Intensität der Verfasser des Promptuariums hinter das Werk gegangen ist und mit wie großer Sicherheit und Zuverlässigkeit die Auskünfte von ihm gegeben werden können. Es ist nicht zuviel gesagt, wenn man feststellt, daß mit dem Promptuarium, das ein riesiges Zettelregister darstellt, jedem zukünftigen Geßner-Forscher ein Arbeitsinstrument zur Verfügung gestellt worden ist, ohne welches eine zuverlässige Forschungsarbeit auf diesem so äußerst komplizierten und vielseitigen Gebiet der Geßner-Forschung kaum mehr ohne unerhörten Zeitaufwand möglich ist.

Zur Illustrierung der Hilfe, welche das Promptuarium dem Forscher bieten kann, wurde im folgenden ein Spezialgebiet, das von Herrn Manfred Peters in Liège bearbeitete, verhältnismäßig kleine Gebiet von Geßners Sprachforschung als Beispiel eines Forschungsplanes hingestellt, zu welchem R.Steiger dem Bearbeiter dieses Gebietes eine Unmenge von Nachweisen zur Verfügung stellen konnte.

Eine große Schwierigkeit für den Geßner-Forscher bildet die Entzifferung der Handschrift. Auch da hat Dr. Steiger keine Mühe gescheut, um dem GeßnerForscher seine Arbeit zu erleichtern. Zur Zeit ist er damit beschäftigt, die zahlreichen, flüchtig hingeschriebenen, meist in Latein verfaßten Notizen auf den in der Universitätsbibliothek Erlangen befindlichen Pflanzenbildern zu entziffern und zu transponieren. Dabei handelt es sich nicht nur um die Schrift Geßners, sondern auch anderer botanisch interessierter zeitgenössischer Forscher, wie Thomas Penny u.a., welche auf den Pflanzenabbildungen ihre Vermerke angebracht haben. 
Im weiteren hat sich Dr. Steiger vorgenommen, nach Verarbeitung des Pflanzenmaterials sich mit den zoologischen Werken Geßners zu befassen, um sie nach den verschiedensten Gesichtspunkten zu registrieren und dadurch der Forschung ebenfalls zugänglich zu machen.

Ich freue mich, den Lesern des Gesnerus den Einblick in diese große Aufschließungsarbeit zu Conrad Geßner vermitteln zu können, nicht zuletzt in der bestimmten Hoffnung, daß der eine oder der andere, der sich bisher gescheut hat, dem «Riesen» Conrad Geßner seine geschichtsforschende Kraft zu widmen, sich durch die uneigennützige Erschließungsarbeit Dr. Steigers ermutigen lasse, dies zu tun. Die große vorbereitende Arbeit Dr. Steigers läßt die Hoffnung aufkommen, es könnte einmal jemand hinter die große Aufgabe gehen, Conrad Geßners Leben und Werk in einer Biographie und Werkgeschichte darzustellen, die des größten Naturforschers und Arztes Zürichs im 16. Jahrhundert würdig sind.

In einem späteren Zeitpunkt hoffe ich, über die weitere Aufschließungsarbeit Dr. Steigers zu Conrad Geßners gewaltigem Werk, das für den einzelnen ohne bibliothekarisch-bibliographische Hilfe nicht überschaubar ist, wieder berichten zu können.

H. Fischer

\section{Jahresbericht 1967, erstattet von Dr. Rudolf Steiger}

Arbeiten: A. Rekonstruktion von Geßners Privatbibliothek

B. Auswertung des botanischen Nachlasses

C. Nachführung des Promptuariums

D. Sammlung von Photokopien

E. Korrespondenz

Beilagen :

I. Autoren der aufgefundenen Handexemplare

II. Verteilung ihrer Werke auf Wissensgebiete

III. Pflanzenspender

IV. Spender nach Pflanzenzahl

V. Herkunftsorte der Pflanzen

VI. Herkunftsorte nach Pflanzenzahl

VII. Promptuarium: Hauptgruppen

VIII. Promptuarium: Hauptgruppe F

IX. Promptuarium: Hauptgruppe N

X. Promptuarium: Fachgruppe Medizin

XI. Pflanzenbild: Photokopie

XII. Pflanzenbild: Transkription der Legenden

XIII a, b. Auskunft an Dissertanden 


\section{A. Rekonstruktion von Geßners Privatbibliothek}

Bis 1964 finden sich in der Literatur meines Wissens nur 4 aus Geßners Besitz stammende Bücher unserer Bibliothek erwähnt.

Während der Vorbereitung zur Geßner-Ausstellung 1965 kamen 11 weitere zum Vorschein.

Die 15 bis dahin bekannten Handexemplare Geßners in der Zentralbibliothek sind aufgezählt in Hans Fischers Geßner-Erinnerungsschrift (Neujahrsbl. Naturf. Ges., Zürich 1966), S. 141.

Im Verlauf einer noch nicht abgeschlossenen systematischen Durchsicht unserer alten Bestände hat sich die Zahl der durch Geßners Hand gegangenen Bücher auf 165 erhöht. Davon sind 61 mit dem Besitzvermerk Geßners versehen, 16 andere wurden Geßner von Freunden gewidmet. 59 enthalten keinen Besitzvermerk, aber andere Einträge von Geßners Hand. Bei 17 ist die Zugehörigkeit zu einem Sammelband in Geßners Besitz (Notizen Geßners auf den Buchdeckeln) der einzige Hinweis. 12 weitere Bücher tragen handschriftliche Widmungen Geßners an Freunde.

Die 153 Bücher (bibliographische Einheiten), die auf Geßners Regalen gestanden haben, enthalten 190 Schriften von 152 Autoren (die verschiedenen Schriften in Sammelpublikationen einzeln gezählt; einige Autoren sind durch mehrere Schriften vertreten). Die Autoren sind in der Beilage I verzeichnet.

In der Verteilung ihrer Schriften auf die einzelnen Wissensgebiete (Beilage II) fällt auf, daß die Astronomie (Kosmographie) verhältnismäßig stark vertreten ist. Bisher haben Anhaltspunkte für eine intensivere Beschäftigung Geßners mit diesem Gebiet gefehlt. Wahrscheinlich hängt sie mit seiner Lehrtätigkeit zusammen.

$\mathrm{Zu}$ den bisher bekannten Handexemplaren eigener Werke sind hinzugekommen:

Historia plantarum. 1541, (D 459 ${ }_{9}$ )

Martialis epigrammata. 1544. (VII 377)

Bibliotheca universalis. 1545. (PA 18)

Auf die besondere Bedeutung der mit zahlreichen Marginalien versehenen Bibliotheca universalis hat Herr Direktor Dr. Paul Scherrer im Librarium 10 (1967) Heft 1 hingewiesen.

Als weitere, mit vielen oder wichtigen Einträgen Geßners versehene Handexemplare sind zu erwähnen:

Apianus. Cosmographicus liber. 1524 (FF 130 a) 
Aristoteles. Opera omnia. 1539 (B 83 \& a)

Aristoteles. Opera omnia. 1542 (XXIII 41, 42)

Avicenna. De animalibus. s.a. $\left(3.16_{5}\right)$

Dodoens. Histoire des plantes. 1557 (Gal XVI 17)

Dodoens. Cruydeboeck. 1563 (I R 2) mit eigenhändigen Pflanzenzeichnungen Geßners

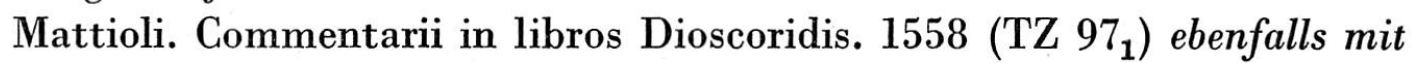
eigenhändigen Pflanzenzeichnungen Geßners

Orpheus. Argonauticon. 1523 (XX 185)

Pausanias. Do tota Graecia libri decem. 1550 (VH 21)

Pindaros. Pythia, Nemea, Isthmia. 1542 (Gal XX 179)

Pollux. Onomasticon. 1520 (IV 78 1 )

Quintus Calaber. Derelictorum ab Homero libri. s.a. (Gal IV 349)

Razi. Liber ad Almansorem. 1497. (3.11 $)$

Regiomontanus. Tabulae directionum. 1490 (Gal II 129 ${ }_{3}$ )

Theophrastos. Sparse de plantis sententiae. s.a. (Gal XXII 10991)

Thevet. Singularitez de la France antarctique. 1558 (L 165)

Xenophon. Opera. 1533 (Dr. Ms 4301)

Der in der Zentralbibliothek erhalten gebliebene Teil von Geßners Bibliothek hat sich bereits als unerwartet groß erwiesen. Eine Fortsetzung der Suchaktion dürfte weitere Funde zeitigen. Es steht aber fest, daß sich wichtige Handexemplare Geßners auch in Basel, Erlangen, London und in den USA befinden. Die Beschaffung von Photokopien ihrer von Geßner stärker beschrifteten Seiten sollte als Zukunftsaufgabe im Auge behalten werden.

\section{B. Auswertung des botanischen Nachlasses}

Die Pflanzenzeichnungen aus Geßners Nachlaß, deren Originale sich in der Universitätsbibliothek Erlangen befinden, enthalten zahlreiche, für Geßners Eigengebrauch flüchtig hingeworfene Notizen.Zum Teil mühsam zu lesen und zu deuten, enthalten sie eine Fülle von Angaben, die Geßners botanische Sammeltätigkeit während der letzten Lebensjahre beleuchten.

Ich habe mir vorgenommen, diese Angaben für das Promptuarium zu verzetteln und damit begonnen, indem ich an Hand unserer Photokopien jede Erwähnung eines Spenders oder eines Fundortes der abgebildeten Pflanzen registrierte. Aus dieser Arbeit sind die nachstehend erwähnten Listen hervorgegangen: 
Die Beilage III enthält die Namen von 137 Personen, die Geßner Pflanzen oder Abbildungen von solchen, manchmal auch nur Samen geschickt haben. Es ist wohl anzunehmen, daß die meisten Sendungen von Briefen begleitet waren. Nur von 37 Spendern haben sich Briefe an Geßner erhalten und in den wenigsten ist von einer Sendung die Rede. Ein neuer Beweis, daß der Großteil von Geßners Korrespondenz verlorengegangen ist oder anderwärts unbeachtet liegt.

Die Liste der mehrfachen Spender (Beilage IV) vermittelt ein unerwartetes Bild von der Intensität des Verkehrs zwischen Geßner und seinen Tauschpartnern. Eine so umfangreiche botanische Beziehung zu Dalechampius und Dourez war z.B. unbekannt. Selbst unter den eifrigen Spendern begegnet man Personen, von deren Zugehörigkeit zu Geßners Freundeskreis man bisher nichts wußte, z.B. Bumannus, Cortusius, Eglin, Scheidenmacher.

Aufschlußreich ist auch die Liste der Pflanzenherkunftsorte (Beilage V). Zunächst erkennt man den geographisch weitgespannten Rahmen von Geßners Sammeltätigkeit: Polen, die Türkei, Ägypten, Afrika und Amerika werden genannt. Die kursiv gesetzten Fundorte hat Geßner selbst besucht. Davon sind erstmals in Erscheinung tretend: Amden, das Bernbiet, Flums, der Gonzen, der Gotthard, Pfäfers, Plurs, der Septimer, Walenstadt und Wesen. Leider reichen die Angaben nicht aus, um das Itinerar von Geßners Bündner Reise von 1561 mit Sicherheit zu bestimmen. Nicht ohne Reiz ist die Zusammenstellung der Fundorte in der Umgebung von Zürich.

Die Verteilung der Pflanzen auf Herkunftsorte (Beilage VI) zeigt wie erwartet, daß Geßner die meisten Pflanzen beim Botanisieren in der Umgebung Zürichs gefunden hat. Diejenigen aus Montpellier hat er wohl alle von Joh. Bauhin erhalten, dessen große Rolle beim Aufbau von Geßners Pflanzensammlung aus den Notizen immer wieder ersichtlich ist. Die Pflanzen aus dem Wallis dürfte Kaspar Collin, diejenigen von Chur Jo.Fabritius geliefert haben. Dagegen hat Geßner die Pflanzen vom Bockmattli, vom Zürichsee, aus der Umgebung von Basel und vom Katzensee wohl fast alle selbst gesucht.

\section{Nachführung des Promptuariums}

Ein als Vorarbeit zur Geßner-Ausstellung 1965 angelegtes Zettelregister wurde nachher zu einem Promptuarium erweitert, das alles in der Zentralbibliothek greifbare, der Geßner-Forschung direkt oder indirekt dienende 
Material nachweisen soll. Außerdem werden darin in der Literatur angetroffene Stellen, die für die Erkenntnis von Geßners Leben und Werk von Belang sind, in extenso, gekürzt, zusammengefaßt oder auch als bloßer Hinweis registriert.

Dieses Promptuarium wurde im Berichtsjahr um etwa 4000 auf etwa 16000 Notizen vermehrt, die, auf etwa 12000 Zetteln vermerkt, in 16 Schachteln aufbewahrt werden.

\section{Einrichtung des Promptuariums}

Es wäre verfrüht, diese Einrichtung bis in alle Details schildern zu wollen, weil sich im einzelnen noch manches ändern kann. Dagegen dürfte sie als Gesamtkonzeption, die sich bewährt hat, bestehen bleiben. Von ihr soll die Rede sein.

Die Systematik wurde so entworfen, daß sie ihre Aufgabe, der ErschlieBung des Geßner-Materials zu dienen, möglichst gut erfüllen kann. Auch wurde darauf gesehen, daß sie eine Erweiterung oder Verfeinerung zuläßt, ohne an Übersichtlichkeit zu verlieren.

Ihre Hauptgruppen sind aus der Beilage VII, der Inhalt der Hauptgruppen $\mathrm{E}$ und $\mathrm{N}$ aus den Beilagen VIII und $I X$, derjenige des Fachgebietes Medizin aus der Beilage $X$ ersichtlich.

Inhalt der übrigen Hauptgruppen:
A. Übersichten :
1. Systematische Übersicht
2. Alphabetische Übersicht
3. Zitierte Literatur
B. Allgemeine Hilfsmittel:
1. Adressen
2. Bibliographien
3. Lexika und Enzyklopädien
4. Wörterbücher
C. Biographische Darstellungen: 1. Autobiographien
2. Selbständige Biographien
3. Biographien in Sammelwerken
4. Aufsätze und Vorträge
5. Ausstellungen
D. Biographische Chronologie: (nach Jahren geordnete Fakten)
[E. Biographische Einzelfragen: Beilage VIII]
F. Briefe :
1. Nachweis
2. Korrespondenten 
G. Handschriften: (nach Standorten geordnet)

H. Drucke: 1. Alphabetisches Verzeichnis

2. Drucker und Verleger

3. Verlagsorte

I. Titelregister der Handschriften und Drucke

K. Handexemplare: 1. Alphabetisches Verzeichnis

2. Standortsverzeichnis

L. Ikonographie: 1. Geßner

2. Lehrer, Freunde, Gönner

3. Aufenthaltsorte

M. Gesneriana außerhalb der Zentralbibliothek: (nach Aufbewahrungsorten)

[N. Fachgebiete: Beilagen IX und X]

O. Personenregister (Lebensdaten, Erwähnungen bei Geßner)

P. Ortsregister (Erwähnungen bei Geßner)

Q. Sachregister (Begriffe wie Münzwesen, Schulwesen, Stipendien)

R. Wortregister (nachgeschlagene Wörter)

S. Abkürzungen und Zeichen (von Geßner in seinen handschriftlichen Notizen gebraucht)

Im Promptuarium sind einige der durch Nummern bezeichneten Untergruppen weitergegliedert. Diese Feingliederung richtet sich nach dem Anfall von Zetteln und befindet sich bis zum Abschluß des Ganzen im Fluß. Im Prinzip wird angestrebt, jeder Notiz einen eng definierten Platz anweisen zu können, an dem sie rasch wieder aufzufinden ist. Wenn eine Angabe an verschiedenen Stellen gesucht werden kann oder für mehrere Zusammenhänge von Wert ist, wird sie an allen diesen Orten mindestens als Hinweis vermerkt.

Die alphabetische Übersicht A.2) verzeichnet die den Leitkartentiteln entnommenen Schlagworte und erschließt dadurch die ganze Systematik bis in ihre feinsten Verästelungen. Dies gestattet eine sichere Einordnung jeder neuen Notiz und ihr leichtes Wiederauffinden.

\section{Sammlung von Photokopien}

Sie soll als Ergänzung des Promptuariums zur Erschließung unseres Geßner-Materials in der Weise beitragen, daß sie es jedem, der sich mit Geßner befassen will, erlaubt, ohne langwieriges Zusammensuchen die ihn interessierende Dokumentation zu überblicken und einen Begriff von deren 
Aussagewert zu bekommen, ohne schon die Originale beiziehen zu müssen. Wertlose Bestellungen und ein unnötiger Verschleiß der Originaldokumente werden dadurch vermieden. Auch dürfte die Sammlung, indem sie den Weg zu Geßner ebnet, ein Ansporn zu vermehrter Beschäftigung mit unserem Gelehrten sein. Sie umfaßt gegenwärtig etwa 4000 Blätter und besteht aus folgenden Abteilungen:

1. Photokopien, die den Inhalt der Geßner-Ausstellung von 1965 in vollem Umfang und ähnlich festhalten, wie dies mit der Ausstellung «Polyhistorie im alten Zürich» geschehen ist. Die Aufnahmen sind alle erstellt und geordnet, doch konnte das Aufkleben derselben auf Untersatzblätter noch nicht besorgt werden.

2. Photokopien welche die obige Abteilung so ergänzen, daß sie als Unterlagen für eine vertiefte Darstellung der Ausstellungsthemen dienen können.

3. Photokopien der Titelblätter und charakteristischer Seiten von Geßners Handexemplaren.

4. Photokopien datierter Schriftproben Geßners, welche die zeitliche Einstufung undatierter Dokumente an Hand der Schriftmerkmale ermöglichen sollen.

5. Photokopien von Schriftproben aus dem Bekanntenkreis Geßners als Hilfsmittel zur Identifizierung unbekannter Schreiber.

6. Photokopien der existierenden Geßner-Porträts und -Wappen, der besten Porträts seiner Lehrer, Freunde und Gönner, von zeitgenössischen Veduten der von Geßner besuchten Orte und von Landkarten, die Geßner benutzt haben könnte.

7. Photokopien der zur Auswertung für das Promptuarium geeigneten Pflanzenbilder aus Geßners Nachlaß. Sie wurden von unserer Sammlung Ms Z VIII 394 \& a-c, 395 kopiert, um diese zu schonen und als Arbeitsinstrument zu dienen. Über eine erste Auswertung der Pflanzenbilder wurde im Abschnitt B dieses Berichtes referiert. Als zweite Arbeit ist eine Transkription von Geßners Legenden zu diesen Pflanzenbildern geplant. Sie verfolgt den doppelten Zweck: 1. eine gründliche Erfassung der biographischen Anhaltpunkte; 2. eine wissenschaftsgeschichtliche Interpretation der Pflanzenbilder zu erleichtern. Von verschiedener Seite ist darauf hingewiesen worden, daß die oft schwierige Entzifferung von Geßners pro domo hingeworfenen Notizen ein Grund ist, weshalb sich unsere paläographisch meist nicht geschulten Botaniker scheuen, sie zu kommentieren. Die Existenz einer Transkription dürfte mithelfen, diese Scheu zu überwinden. Solange man sich bei uns damit begnügt, Geßner von ferne zu bewundern, 
müssen wir gewärtig sein, von ausländischen Stimmen an eine nationale Aufgabe erinnert zu werden. Ein deutscher Botaniker hat mir kürzlich geschrieben: «Ich habe bei meinen Kollegen mit den Kopien einen großen Eindruck gemacht. Geßners botanische Leistung ist ja weithin unbekannt! Ich glaube, es wäre eine Kulturtat ersten Ranges, wenn jemand sich entschließen könnte, das gesamte Manuskript herauszugeben. Wie denken Sie darüber? Ob die Schweizer Botaniker hier nicht eine Pflicht zu erfüllen hätten?»

\section{Erläuterung eines Pflanzenbildes}

Die Beilagen XI und XII und deren nachstehende Interpretation möchten an einem Beispiel zeigen, welcher Aussagewert den Pflanzenbildern Geßners zukommt.

Zunächst ist hervorzuheben, daß beide Pflanzen mit einer Lebensnähe dargestellt sind, die selbst von modernen Pflanzenabbildungen selten erreicht wird. Unsere stark verkleinerte Abbildung vermag zwar die Schönheit von Geßners Originalaquarell nicht wiederzugeben, aber die botanischen Merkmale treten auch hier so gut in Erscheinung, daß es keine Mühe macht, selbst die Spezies zu bestimmen.

Bei der Scutellaria galericulata (Pflanze links) fragt sich Geßner, ob sie mit einer Pflanze identisch sei, die Aldrovandi zu Unrecht als eine Species der Gratiola bezeichne. Offenbar hat Geßner von Aldrovandi eine Pflanzenabbildung erhalten, die er nun mit der seinen vergleicht. Die Gattung Scutellaria unterscheidet sich von der Gattung Gratiola fast nur dadurch, daß sie einen viereckigen statt einen runden Stengel aufweist. Auf dieses Merkmal der Scutellaria weist Geßner ausdrücklich hin (caulis quadrangulus est).

Weil die Pflanze noch keinen Namen hat, möchte er sie nach der sattelförmigen Gestalt ihrer Samenkapseln «Ephippiaria» nennen. Sein zweiter Vorschlag geht dahin, sie nach der Ähnlichkeit ihres Geruches und Geschmackes mit dem Andorn «Ballote palustris» (Sumpfandorn) zu taufen. Die Auffassung, daß gleicher Geruch und Geschmack auf Verwandtschaft hindeute, ist typisch für den Arzt und Pharmakologen Geßner. Das Nomen Ballote palustris ist ein frühes Beispiel für die Anwendung einer binären Nomenklatur, die sich bekanntlich erst seit Linné durchgesetzt hat.

Die Utricularia neglecta (rechts) nennt Geßner eine Pflanze ohne Wurzel (herba sine radice). Somit scheint er deren untere Fortsetzung bereits richtig als Rhizom und deren scheinbare Fasern als gefiederte Blätter aufzu- 
fassen. Die Bläschen der letzteren, deren Zweck als Fangblasen er noch nicht erkennen konnte, erscheinen ihm so charakteristisch, daß er sie zweien seiner Namenvorschläge (Lentibullia vel Lentibullaria) zugrunde legt.

Obschon Geßner die beiden Pflanzen als erster dargestellt und beschrieben hat, sind die von ihm vorgeschlagenen Namen zufolge der Nichtveröffentlichung seines Pflanzenwerkes als Artbezeichnungen untergegangen. Einzig Lentibullaria (worauf mich Herr Dr. CAsper, Jena, aufmerksam machte) ist auf merkwürdigen Umwegen und sprachlich verdorben in die Bezeichnung der ganzen Pflanzenfamilie (Lentibulariaceae) übergegangen.

Geßner scheint als erster Botaniker Wert darauf zu legen, die Blütezeit seiner Pflanzen anzugeben, so sagt er von der Scutellaria, daß er sie am 10.August, von der Utricularia, daß er sie weder am 13. Juni noch am 10. August blühend angetroffen habe.

Die Hinweise auf andere Pflanzen lassen das Bestreben erkennen, mit falschen Identifizierungen aufzuräumen, bloß Ähnliches zu trennen und Verwandtschaftsbeziehungen aufzudecken; ihre knappe Formulierung, zum Teil als Frage, läßt aber auch erahnen, daß er erst am Anfang dieser gewaltigen Arbeit stand.

Die Art, wie Geßner Aldrovandi erwähnt, läßt vermuten, daß er von diesem Gelehrten (A.Ulysses, 1522-1605, Professor der Medizin in Bologna, später Gründer des botanischen Gartens daselbst) entweder Pflanzen oder Abbildungen von solchen erhalten hat. Genaueres darüber ist nicht bekannt, weil die Briefe Aldrovandis an Geßner verschollen sind. Ob sich die Briefe Geßners in Bologna erhalten haben, wäre zu erkunden.

Als Finderin der Utricularia ist Geßners Frau genannt, die richtig beobachtete, daß es sich um eine untergetaucht wachsende Pflanze handelt, von der einzig der Blütenstengel über das Wasser ragt. Das Urteil über diese Frau, von der man bisher nur wußte, daß sie «immer krank und eine schlechte Haushälterin » war, dürfte zu revidieren sein.

Die Gegend des Katzensees wird als Fundort beider Pflanzen bezeichnet. Vielleicht war sie schon damals als «botanisches Schatzkästlein » bekannt (wie Diethelm Fretz sich ausdrückt). Außer botanisierenden Gelehrten waren daselbst wohl «Kräuterweiblein» anzutreffen, die nach Heilpflanzen suchten. In Begleitung solcher mag Frau Dr. Geßner den Katzensee aufgesucht und daselbst die blühende Utricularia gefunden haben. Geßner hat sie nicht blühend «in den hiesigen Gräben zu den Drei Königen » angetroffen. Dieser Fundort war vor der damaligen Stadtmauer, etwa in der Gegend des heutigen Pestalozzidenkmals gelegen. 
In der Flora der Schweiz von Schinz und Keller ist die Utricularia neglecta nicht erwähnt. Sie dürfte also nach Geßners Zeit als wildwachsende Pflanze bei uns verschwunden sein. Vielleicht weil sie (wie ich lese) vom Wassergeflügel gern gefressen wird, vielleicht auch, weil sie als Heilkraut und als botanisches Kuriosum gefragt war. Wir hätten also ein botanisches Gegenstück zum berühmten Waldrapp vor uns: beide sind heute bei uns ausgestorben. Eine Erkenntnis, die wir Geßner verdanken.

\section{E. Korrespondenz}

Alle an die Zentralbibliothek oder mich gerichteten Anfragen, die Geßner betreffen, werden erschöpfend beantwortet, und diese Korrespondenz wird als Bestandteil der Geßner-Dokumentation aufbewahrt.

Während des Berichtsjahres gingen Auskünfte (zum Teil mehrere) an folgende Herren:

Dr. Jost KASPER, Limnologe, Jena-Lobeda

Dr. Fichtner, Assistent, Medizingeschichtliches Institut, Tübingen

Prof. Dr. Hans Fischer, Zollikon

Herr C. Gesner v. D. Voort, Haag

Herr C.-E. Kohlhauer, Ansbach

Prof. Dr. Friedr. MARKgraf, Botanischer Garten, Zürich

Herr Manfred Peters, Licencié, Liège

Dr. ERNST FURRER, Botaniker, Zürich

Dr. Charles SalzmanN, Rüschlikon

Herr ERNST KüNZLI, Zürich

Herr Max HofmanN, Art. Inst. Orell Füßli, Zürich

Eine unter voller Auswertung des Promptuars erstellte Auskunft ging an Herrn Manfred Peters (Beilagen XIII $a, b$ ).

18. 1.68

R. Steiger 
Autoren in Geßners Privatbibliothek

(in Klammern: zwei oder mehr Werke und Ausgaben)

Aelianus

Aelius Spartianus

Aischylos

Albumasar

Alciatus

Alexander Aphrod.

Alexander Trallean.(2)

Alfonsus Hispal.

Amatus Lusit.

Apianus

Apicius

Apollinaris

Apollodoros

Aretaios

Aristophanes

Aristoteles (2)

Arrianus

Athenagoras (2)

Avicenna

Barbarus

Bayfius

Bibliander

Boltz

Brasavolus (2)

Budaeus

[Buechlin, ein]

Calcagnini

Camerarius

Capitius

Capito

Cardanus

Chalkondylas

Chrysoloras

Cisnerus
Conteremus

Cordus

[Cosmographia]

Cotta

Curio

[Decalogus]

Didymos (2)

Dio Chrysostomos

Dionysios Alexandr.

Dionysios Apher

Dodoens (2)

Dryander

Egenolphus

Elias Judaeus

Eukleides

Eustathios (4)

Flaminius

[Florilegium]

Fuchsius

Galenos (2)

Geßner (7)

Glareanus

Gordonius

Goupylius

Guido de Cauliaco

Hesiodos

Hesychios

Hieronymus de Ferr.

Hippocrates

Homeros

Honterus (2)

Huttenius

Johannes fil. Mesue

Johannes Gramm.
Johannes Saxon.

Justinus Philos.

Juvenalis

Kimchi

Lacisius

Lazius

Lemnius

Leupoldus Ducis Austriae fil.

Lukianos

Lykophron

Macrobius

Manardus

Manilius

Martialis

Massa

Matthiolus (2)

Melanchthon

Michael Ephesios

Micyllus

[Mikropresbytikon]

Mirandula

Montuus

Morsianus

Münster

Nicolaus Alexandr.

[Novum Testamentum]

Oppianos

Oreibasios

Orpheus

Ovidius (2)

Paulus Aegineta

Pausanias

Persius 


$\begin{array}{lll}\text { Petronius } & \text { [Recept, ein] } & \text { Theophrastos } \\ \text { Petrus Ranzanus } & \text { [Regiment, ein] } & \text { Thevet } \\ \text { Phrygio } & \text { Regiomontanus } & \text { Thukydides } \\ \text { Picardus } & \text { Rota } & \text { Thurinus (4) } \\ \text { Pindaros (2) } & \text { Sambucus (2) } & \text { Thylesius } \\ \text { Pintianus } & \text { [Scriptores gnomici] } & \text { Tzetzes (2) } \\ \text { Platina } & \text { Simplicius } & \text { Vadianus } \\ \text { Plato } & \text { Ruphos Ephesios } & \text { Valleriola } \\ \text { Plautus } & \text { Sextus Philos. } & \text { Velsius } \\ \text { Plutarchos } & \text { Sophokles } & \text { Vipera (3) } \\ \text { Politianus } & \text { Statius } & \text { [Vitae Caesarum] } \\ \text { Pollux (2) } & \text { Stephanos Byzant. } & \text { Vitalis de Furno } \\ \text { Porphyrius } & \text { Suetonius } & \text { Welcurio } \\ \text { Prokles } & \text { Sylvius (4) } & \text { Willichius } \\ \text { Ptolemaios }(2) & \text { Synesios } & \text { Xenophon } \\ \text { Quintus Calaber } & \text { Tacitus (4) } & \text { Zacharia } \\ \text { Razi } & \text { Themistios Euphr. } & \end{array}$

Beilage II

Verteilung der Werke auf Wissensgebiete

$\begin{aligned} 59 & \text { Philologie } \\ 43 & \text { Medizin } \\ 15 & \text { Astronomie } \\ 13 & \text { Philosophie } \\ 11 & \text { Zoologie } \\ 10 & \text { Theologie } \\ 9 & \text { Geschichte } \\ 8 & \text { Botanik } \\ 6 & \text { Lexikographie } \\ 5 & \text { Varia }\end{aligned}$

3 Naturwissenschaft im allgemeinen

2 Streitschriften

1 Alchemie

1 Bibliographie

1 Farbenkunde

1 Jurisprudenz

1 Mathematik

1 Numismatik

1 Politik

Bemerkung zu den Beilagen I und II

Da sich in beiden Listen nur der bisher ermittelte Bestand von Geßners Privatbibliothek widerspiegelt, ist ihre Aussage nicht absolut. Sie dürfte aber, indem sie einen ersten Eindruck vermittelt, von Nutzen sein. 


\begin{tabular}{|c|c|c|}
\hline Aberli Jo. & Du Choul Jo. & Korsten \\
\hline Aemylius Ge. & Dunus Thadd. & Krafftheim Crato v. \\
\hline Aldrovandi Ulyss. & Eglin Thob. & Kreichius Joach. \\
\hline Anguillara Aloys. & Eschenreutterus Gall. & Landenbergius J. Rod. \\
\hline Aretius Bened. Mart. & Fabritius Sebast. & Landgravius (?) \\
\hline Bauhinus Jo. & Fabritius Montanus Jo. & Lemnius Conr. (?) \\
\hline Baumann Gerh. & Fontanonus Franc. & Leo Jac. \\
\hline Bechius Phil. & Forerus Conr. & Leonorus Adam. \\
\hline Belicoccus Andr. & Fuggerus Phil. & Lindinner \\
\hline Bellonius Petr. & Fuggerus Ulr. & Lonicerus Adam. \\
\hline Bodenstein Adam v. & Fulgonus Jac. & Lucas (Vorname?) \\
\hline Boutin Petr. & Funccius Jac. & Maranta Barthol. a \\
\hline Bullinger Hch. (Sohn) & Funccius Jo. & Marius (Vorname?) \\
\hline Bumannus Jac. & Garnerius & Massarius Hier. \\
\hline Caius Jo. & Gassarus Achilles & Matthiolus Andr. \\
\hline Calceolarius Franc. & Geßner (Gattin) & Moderatus Jul. \\
\hline Camerarius Joach.(fil.) & Geßner Hch. & Montisaurus Natal. \\
\hline Campellus & Ghinus Luc. & Mundella Alois. \\
\hline Cardanus Hieron. & Goeldlinus Joach. & Muraltus Alois. \\
\hline Cellarius Isaacus & Gratarolus Guilh. & Murer Jos. \\
\hline Chortander Adr. & Großmannus & Nueschelerin \\
\hline Clauser Ge. & Guilandinus Melch. & Obrecht Didymus \\
\hline Clauser Nic. & Hafner Petr. & Occo Ad. \\
\hline Clusius Car. & Hallerus Wolfg. (?) & Ollingerus Paul. \\
\hline Coldenbergius Petr. & Hawenreutterus Sebald. & Oppenheimius Jac. \\
\hline Collinus Casp. & Herculanus (Vorn. ?) & Parkhurstius Jo. \\
\hline Constantinus Rob. & Herling & Pena Petr. \\
\hline Conzadini & Heroldus Hier. & Peyerus Alex. \\
\hline Cortusius Jac. & Heroldus Jo. & Platterus Fel. \\
\hline Dalechampius Rob. & Hiel Laur. & Pontisella Jo. \\
\hline Delker Jo. & Huberus Chrysosth. & Praun Balth. \\
\hline Dodonaeus Remb. & Jacobus Petr. & Prenosius (?) Jo. \\
\hline Donzellinus Hier. & Joubertus Laur. & Quadri \\
\hline Dourez Valerand. & Kentmannus Jo. & Quercetanus Eusth. \\
\hline
\end{tabular}




\begin{tabular}{|c|c|c|}
\hline Rassius Nicol. & Schwyzer Christoph. & Sylvester Isaacus \\
\hline Reginaldus Jac. & Sebastianus (Vorn.) & Toxites Mich. \\
\hline Rheticus Joach. & Secundus Caelius & Turnerus Guilh. \\
\hline Rihelius & Seebach Georg & Ursinus Zach. \\
\hline Rondeletius Guilh. & Segesseria (moniale) & Vergerius Petr.Paul. \\
\hline Rotacherus Christoph. & Sighartus Ge. & Wellenbergin \\
\hline Salvianus Hippol. & Simlerus Josua & Wirsungus Phil. \\
\hline Schaerer Marc. (?) & Sittardus Ge. & Wolphius Casp. \\
\hline Schaererin & Solarius Hugo & Wonlichius Rod. \\
\hline Scheidenmacher Lud. & Solonander & Württemberger (?) Jo \\
\hline Schmidlappius Jo. & Stephanus Carol. & Zimmermann \\
\hline Schneeberger Ant. & Sturm & Zwinger Theod. \\
\hline
\end{tabular}

Beilage IV

Die Spender nach der Anzahl der gelieferten Pflanzen

$\begin{aligned} 51 & \text { Dalechampius } \\ 41 & \text { Dourez } \\ 29 & \text { Bauhinus } \\ 29 & \text { Kentmannus } \\ 27 & \text { Coldenbergius } \\ 24 & \text { Calceolarius } \\ 19 & \text { Forerus } \\ 14 & \text { Fabritius Montanus } \\ 12 & \text { Aldrovandi } \\ 9 & \text { Aemylius } \\ 8 & \text { Bumannus } \\ 8 & \text { Camerarius } \\ 8 & \text { Occo } \\ 7 & \text { Cortusius } \\ 6 & \text { Eglin } \\ 6 & \text { Ollingerus } \\ 6 & \text { Scheidenmacher } \\ 6 & \text { Schmidlappius } \\ 5 & \text { Aretius } \\ 5 & \text { Guilandinus }\end{aligned}$

$\begin{array}{ll}5 & \text { Hafner } \\ 5 & \text { Heroldus } \\ 5 & \text { Hiel } \\ 5 & \text { Schneeberger } \\ 5 & \text { Zwinger } \\ 4 & \text { Belicoccus } \\ 4 & \text { Clusius } \\ 4 & \text { Gratarolus } \\ 4 & \text { Korsten } \\ 4 & \text { Mundella } \\ 4 & \text { Pontisella } \\ 3 & \text { Bellonius } \\ 3 & \text { Caius } \\ 3 & \text { Funccius, Jo. } \\ 3 & \text { Hawenreutterus } \\ 3 & \text { Oppenheimius } \\ 3 & \text { Platterus } \\ 3 & \text { Rassius } \\ 3 & \text { Reginaldus } \\ 3 & \text { Secundus }\end{array}$

3 Wolphius

2 Baumann

2 Collinus

2 Constantinus

2 Dodonaeus

2 Dunus

2 Fabritius Jo.

2 Geßner (Gattin)

2 Huberus

2 Jacobus

2 Kreichius

2 Leo

2 Marius

2 Muraltus

2 Prenosius(?)

2 Sebastianus

2 Sighartus

2 Stephanus

2 Toxites

(Alle übrigen Spender sind als solche nur bei einer einzigen Pflanze erwähnt. Bei manchen Pflanzen dürfte Geßner vergessen haben, den Spender zu erwähnen. Auch ist wahrscheinlich etwa ein Drittel von Geßners Pflanzenzeichnungen verlorengegangen.) 
Fund- oder Herkunftsorte von Geßners Pflanzen (an den kursiv gesetzten Orten hat Geßner selbst botanisiert)

\begin{tabular}{lll} 
Aarau & Davos & Königsberg \\
Afrika & Dübendorf & Kyburg \\
Agde (Provence) & Eglisau & Lägern \\
Aix (Provence) & Eigental & Laufenburg \\
Albis & Engadin & Lausanne \\
Altstetten & Engelberg & Liestal \\
Alvaneu GB & England & Ligurien \\
Amden & Flums & Lindau \\
Amerika & Frankfurt am Main & Lissabon \\
Antwerpen & Frankreich & Lyon \\
Arles & Fribourg & Marseille \\
Augsburg & Gemmi & Mecheln (Belgien) \\
Avignon & Generoso, Mte. & Memphis (Ägypten) \\
Baden und Umgebung & Genf & Moerlingen \\
Baldo, Mte. (bei Verona) & Genfersee & Montpellier und Um- \\
Barten, S. (wo ?) & Glärnisch & gebung \\
Basel und Umgebung & Glarus & Neapel \\
Bellinzona & Glatt & Niederdeutschland \\
Bendlikon & Glattbrugg & Nürnberg \\
Bergamo & Gonzen & Oberhausen zH \\
Bergell & Gotthard & Österreich \\
Bern & Graubünden & Padua \\
Bernbiet & Greifensee & Paris \\
Bockmattli sz & Grenoble & Pavia \\
Bologna & Grifflen vs & Pfäfers \\
Bormio & Haslerberg & Pilatus \\
Braulio, Mte. (bei & Hellespont & Pisa \\
Bormio) & Immensee & Plurs (bei Chiavenna) \\
Breslau & Innsbruck & Polen \\
Calanda & Istrien & Prättigau \\
Chiavenna & Italien & Provence \\
Chur & Kärnten & Rigi \\
Comersee & Kantabrien & Rimini \\
Corsica & Katzensee & Rom \\
& & \\
\hline & &
\end{tabular}




$\begin{array}{lll}\text { Rottweil } & \text { Sitten } & \text { Unterwalden } \\ \text { Savoyen } & \text { Solothurn } & \text { Uster } \\ \text { Scala, Mons (Alvier?) } & \text { Spanien } & \text { Uttigen BE } \\ \text { Schaffhausen und Um- } & \text { Straßburg } & \text { Venedig } \\ \text { gebung } & \text { Susch } & \text { Verona } \\ \text { Schlesien } & \text { Syrien } & \text { Vogesen } \\ \text { Schorndorf (Württem- } & \text { Talwil } & \text { Walensee } \\ \text { berg) } & \text { Teglio (Veltlin) } & \text { Walenstadt } \\ \text { Schuls } & \text { Terretium (Adria) } & \text { Wallis } \\ \text { Schwamendingen } & \text { Thüringen } & \text { Weesen } \\ \text { Schwyz und Umgebung } & \text { Thun } & \text { Winterthur } \\ \text { Sebenico (Dalmatien) } & \text { Tirano } & \text { Zollikon } \\ \text { Septimer } & \text { Tübingen } & \text { Zürich und Umgebung } \\ \text { Siegental BE } & \text { Türkei } & \text { Zug } \\ \text { Sihl } & \text { Ulm } & \\ \text { Silberen sz } & \text { Ungarn } & \end{array}$

Fundorte in Zürich und Umgebung

$\begin{array}{lll}\text { Büelers Hölzlin } & \text { Käferberg } & \text { Seefeld } \\ \text { Drei Königen, Zu den } & \text { Käferholz } & \text { Selnau } \\ \text { Fraumünstergarten } & \text { Klus } & \text { Spitalerwiesen } \\ \text { Friedhof (Groß- } & \text { Kolbenhof } & \text { Spitalerwiesen,Hinder- } \\ \text { münster) } & \text { Limmat } & \text { Spittelmühle } \\ \text { Geißberg } & \text { Lindenhof } & \text { Stadtmauern } \\ \text { Geißbühl } & \text { Manegg } & \text { Uetliberg } \\ \text { Hirschengraben } & \text { Neue Bleike } & \text { Wasserkirche } \\ \text { Hottingen } & \text { Paradiesmühle } & \text { Wollishofen } \\ \text { Im Hardt } & \text { Sankt Gilgen } & \text { Zürichsee } \\ \text { Jonen Müly } & \text { Schipfe } & \end{array}$




\section{Verteilung der Pflanzen auf Fund- oder Herkunftsorte (nach Anzahl der Pflanzen geordnet)}

73 Zürich und Umgebung
43 Montpellier und
Umgebung
17 Bockmattli
16 Wallis
13 Chur
13 Katzensee
12 Basel und Umgebung
10 Davos
8 Augsburg
8 Glatt
7 Bormio
7 Braulio, Mte.
7 Schwyz und Umgebung
6 Bologna
6 Graubünden
6 Lägern
6 Schaffhausen und
Umgebung
6 Weesen
5 Amden
5 Engadin

5 Marseille
5 Pilatus
4 Antwerpen
4. Baldo, Mte.
4. Gotthard
4 Istrien
4 Lausanne
4 Padua
4 Pisa
4 Verona
3 Baden und Umgebung
3 Bernbiet
3 Generoso, Mte.
3 Genf
3 Glarus
3 Gonzen
3 Lyon
3 Nürnberg
3 Prättigau
3 Rigi
3 Sihl

2 Albis

2 Bellinzona

2 Bendlikon

2 Bergamo

2 Chiavenna

2 Greifensee

2 Ligurien

2 Niederdeutschland

2 Oberhausen

2 Österreich

2 Paris

2 Savoyen

2 Septimer

2 Spanien

2 Straßburg

2 Teglio

2 Tirano

2 Unterwalden

2 Venedig

2 Walenstadt

2 Winterthur

(Alle übrigen Fund- oder Herkunftsorte sind nur durch je 1 Pflanze vertreten.)

\section{Hauptgruppen des Promptuariums}

Beilage VII
A. Übersichten
K. Handexemplare
B. Allgemeine Hilfsmittel
L. Ikonographie
C. Biographische Darstellungen
M. Gesneriana außerhalb der Zentral-
D. Biographische Chronologie bibliothek
E. Biographische Einzelfragen
N. Fachgebiete
F. Briefe
O. Personenregister
G. Handschriften
P. Ortsregister
H. Drucke
Q. Sachregister
I. Analytisches Titelregister der Hand-
R. Wortregister schriften und Drucke
S. Abkürzungen und Zeichen 


\section{Promptuarium}

\section{Hauptgruppe E: Biographische Einzelfragen}

Bisherige Schlagworte

Aberglauben

Anlagen, geistige

Arabisch

Arbeitstechnik

Basel, Beziehungen zu

Bergreisen

Bergwelt, Verhältnis zur

Berufungen

Besucher (s.a. Gastlichkeit)

Bibliotheken

Bittschriften

Charakter, allgemeiner

Deutsch

Dienstboten

Ehe

Einkommen

Eklektizismus

Epitaphien

Epithete

Familiensinn

Famuli

Feinde (s.a. Widerstände)

Freigebigkeit

Freunde

Freundestreue

Frömmigkeit (s.a. Religion)

Frühreife

Gastlichkeit (s.a.Besucher)

Geburtsdatum

Gedichte

Gesundheitszustand (s.a.Krankheiten)

Gönner
Grab

Griechisch

Hebräisch

Holländisch

Humanismus

Informationsquellen

Krankheiten (s.a.Gesundheitszustand)

Kriegsereignisse

Kritik

Kurzsichtigkeit

Lateinisch

Lehrer

Leitsätze

Moral

Museum

Mutter

Nachfolger

Nachlaß

Nachrufe

Nachtarbeit

Physische Erscheinung

Pläne

Privatbibliothek

Pseudonyme

Reich, Verhältnis zum

Reisen

Religion (s. a. Frömmigkeit)

Schriftproben

Schüler

Schulbücher

Schuljahre

Sprachkenntnisse im allgemeinen 
Stammbücher

Stil

Stipendien

Testamente

Tod

Todesgedanken

Toleranz

Universalität

Überlastung

Urteile über Geßner
Vaterland, Verhältnis zum

Vergeßlichkeit

Verwandte

Vorgänger

Vorläufer

Wappen

Widerstände gegen Geßner (s.a.Feinde)

Wohnorte

Würdigung (s.a.Urteile)

(s.a. Würdigung)

(Zahl und Wahl der Schlagworte werden nach Gutdünken verändert.)

\section{Promptuarium}

Hauptgruppe N: Fachgebiete

$\begin{array}{lll}\text { Alpinismus } & \text { Lexikographie } & \text { Philologie } \\ \text { Astronomie } & \text { Mathematik } & \text { Philosophie } \\ \text { Bibliographie } & \text { Medizin } & \text { Physik } \\ \text { Bibliothekswesen } & \text { Meteorologie } & \text { Seismologie } \\ \text { Botanik } & \text { Metrologie } & \text { Theologie } \\ \text { Fossilienkunde } & \text { Milchwirtschaft } & \text { Veterinärmedizin } \\ \text { Geographie } & \text { Mineralogie } & \text { Zoologie } \\ \text { Geschichte } & \text { Naturwissenschaft im } & \\ \text { Lehrtätigkeit } & \text { allgemeinen } & \end{array}$




\section{Promptuarium}

Fachgebiet Medizin

(als Beispiel)

Allgemeine Hilfsmittel:

Allgemeine Medizingeschichte:

Geßner als Arzt:

Medizinische Korrespondenz Medizinische Handschriften

Medizinische Drucke

Medizinische Handexemplare

Spezialgebiete:

Diverse Krankheiten:

Einzelfragen:
Bibliographien

Wörterbücher und Lexika

Handbücher

Literatur

Notizen

Allgemeines
Privatpraxis
Stadtarzt

Anatomie

Balneologie

Chirurgie

Epidemiologie

Frauenkrankheiten

Ophthalmologie

Orthopädie

Pharmazeutik

Psychiatrie

Bruchleiden

Epilepsie

Krampfadern

Kropf

Alchemie

Astrologie

Avicenna

Empirie

Galen

Iatrochemie

Iatromathematik

Magie

Selbstversuche

Tierversuche 


\section{zu: Scutellaria galericulata L. (Pflanze links)}

Vide inter Gratias Dei Aldrovandi

Scordio (puto) aliqui cognatum

faciunt, non rectè.

Herba gemellis floribus coeruleis bimm

Katzensee. an Gratiole

species (licet immeritò) Aldrovandi

(Diversa ab hac est 204b.

Ephippiaria vocetur?

Vascula seminum

veluti

ephippta sunt.

Nascitur etiam cubitalis

et ultra.

Augusti decimo florebat.

Caulis quadrangulus et

cavus est.

(Ballote palustris fortè

vocari potest, odore enim et

sapore nonnihil Balloten vel

Urticam marinam refert.

partim subamara, partim

subdulcis. Calida et sicca in

secundo [gradu].

zu: Utricularia neglecta Lehm. (Pflanze rechts)

Herba sine radice. Uxor invenit, in fossis iuxta Lacum Felium. Cauliculus cum floribus eminebat, herba latebat in aqua.

Phacoides palustre. Fictio.

Lentibullia, vel

Lentibullaria. $\}$ Fictio

Vide in Valerii Cordi

in Dioscoridem annotationibus

(in libro 4. capitulo 87. de

Stratiote aquatico.)

etc. $\ddot{\omega} \varrho \alpha[?]$.

Crescit etiam in

Bemerkung:

fossis Trium Regum hîc.

Beide Pflanzen

(Junii 13. non florebat

wurden von

Walo Koch

nec Augusti decimo?

bestimmt.

¿ Zeichen, dessen Bedeutung mir unbekannt ist und das vielleicht nicht von $\mathrm{Geßner}$ stammt. 
Beilage XII

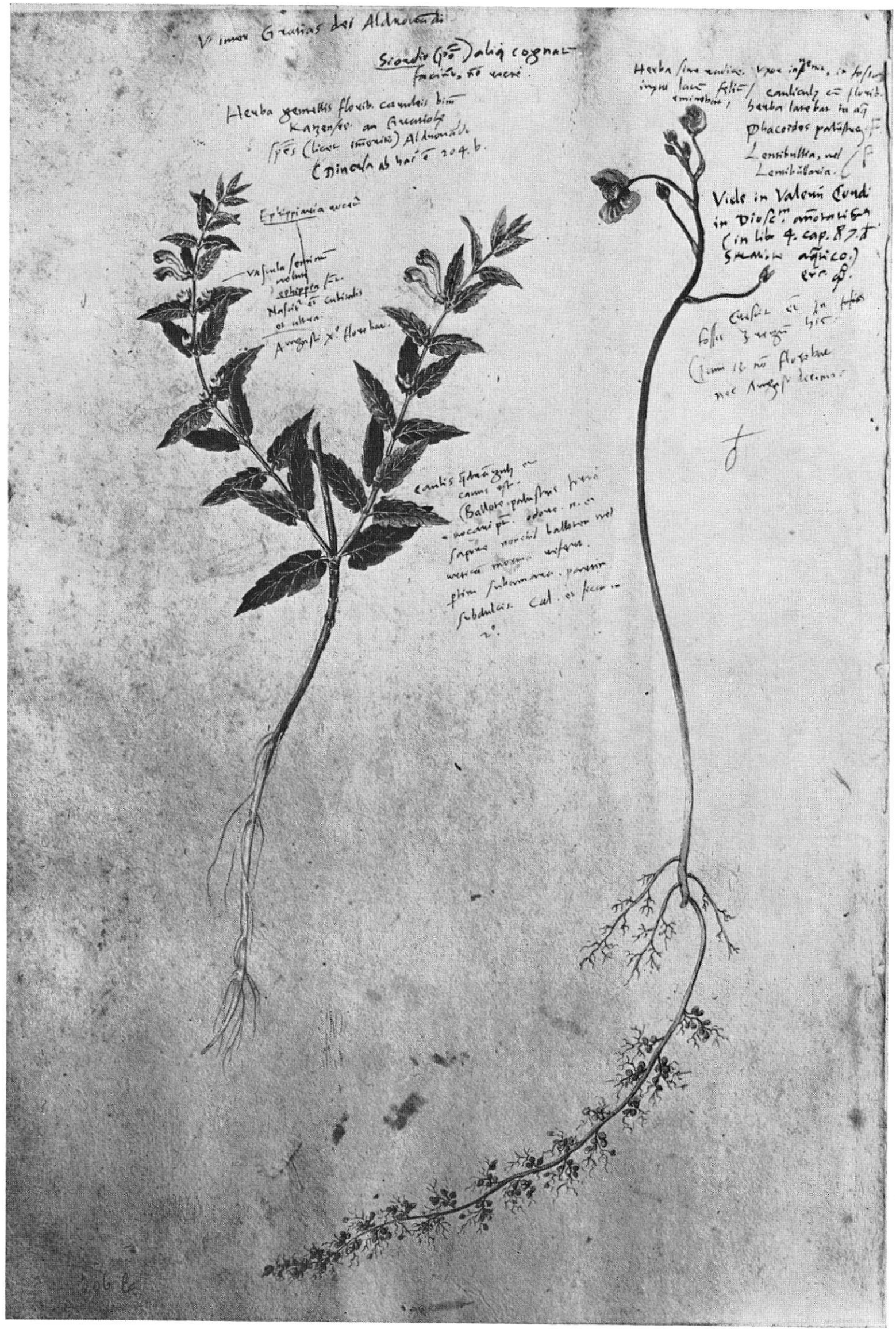

Scutellaria galericulata L. (links) und Utricularia neglecta Lehm. (rechts). Von Konrad Geßner beschriftetes und vermutlich von ihm selbst gezeichnetes Blatt. Original (Aquarell) in der Universitätsbibliothek Erlangen (Ms 2386). ${ }^{2} / 3$ natürlicher Größe 
Manfred Peters

Beilage XIIIa

Licencié en philologie germanique

Assistant aux Facultés Universitaires de Namur

21, rue Saint-Martin

Namur (Belgien)

Herrn Dr. Rudolf Steiger

Zentralbibliothek Zürich

Zürich (Schweiz)

Sehr geehrter Herr Doktor!

Da ich augenblicklich an einer Dissertation über Conrad Geßner arbeite, habe ich mich an den Direktor der Zentralbibliothek gewandt, der mir freundlicherweise wertvolle Hinweise gegeben hat.

Meine Arbeit, die das Wirken Geßners als Linguist und Germanist zum Thema hat, wird folgende Kapitel umfassen:

1. Kurze Vita mit besonderem Bezug auf seine Entwicklung zum Sprachgelehrten.

2. Geßners sprachliche Auffassungen an Hand des Mithridates und des Vorwortes zu Josua Maalers Wörterbuch.

3. Geßners Kontakt mit den sprachlichen Bewegungen seiner Zeit. Sein Verhältnis zu den Buchdruckern. Sein Einfluß auf die späteren Wörterbücher (besondere Berücksichtigung von Frisius, Cholinus, Froschauer).

4. Geßners sprachliches Material:

a) Die verschiedenen Werke, in denen er die deutsche Sprache gebraucht.

b) Woher hat er sein sprachliches Material? Fragenbogenmethode?

c) Die Übersetzungen seiner Werke. Wann sind sie entstanden? Hat er die Übersetzungen selbst überwacht?

d) Seine Sprache.

Vom 22. Mai bis zum 8. Juni werde ich in Zürich selbst arbeiten. Ich wäre Ihnen sehr verbunden, wenn Sie mir bei dieser Gelegenheit eine kurze Unterredung gestatten würden.

Ich danke Ihnen im voraus für Ihre freundlichen Bemühungen und verbleibe

mit vorzüglicher Hochachtung (sig.) Manfred Peters

Bemerkung: Wir drucken den obigen Brief ab, weil sich unsere nachfolgenden Hinweise auf die darin erwähnten Kapitel beziehen. 


\section{Hinweise für Herrn Manfred Peters, Universität Namur, betr. Konrad-Geßner-Material in der Zentralbibliothek Zürich zum Thema «Geßner als Linguist und Germanist»}

Kennen Sie die Arbeiten:

(1) Bächtold, Jak. Die Verdienste der Zürcher um die deutsche Philologie u. Literaturgesch. 1880. (ZB: 1880/6).

(2) Metcalf, G. J. The views of Conrad Gesner on language. (Studies in Germanic languages and literatures pp. 15-26). Washington University Press 1963. (ZB: DL 2141).

(3) Metcalf, G. J. Konrad Gesner's views on the Germanic languages. (Monatshefte für dt. Unterricht, dt. Sprache u. Literatur. 55, S. 149-156). Madison 1963. (ZB: DK 2174).

Ich nehme an, daß Ihnen die einschlägigen Abschnitte in folgenden Werken bekannt sind:

(4) Simler, Josias. Vita Conradi Gesneri. 1566. p. 7r. (ZB: 5. 1655).

(5) Hanhart, Joh. Conrad Geßner. 1824. S. 201-207. (ZB: IV O 486).

(6) Fischer, Hans. Conrad Geßner. (Neujahrsbl. Natf. Ges. Zürich. 168, 1966). S. 72-77. (ZB: KK 562, 168).

(7) Geßner, Conrad; Universalgelehrter, Naturforscher, Arzt. Zürich, Orell Füßli 1967. S. 67, 76-87. (ZB: AX 1320). Im folgenden zitiert als Geßner-Gedenkband.

Sicher haben Sie Kenntnis von der Darstellung bei:

(8) Raumer, Rud. Geschichte der german. Philologie. 1870. S. 41-46. (ZB: IV 0139 f).

Die wichtigste autobibliographische Darstellung Geßners (bis zum Sept. 1562 reichend) ist :

(9) Geßner, Kd. De libris a se editis epistola ad Guil. Turnerum. 1562. (ZB: 5. 334 $4_{2}$ ).

Ein verbesserter, um nachträgliche Drucke und hinterlassene Schriften vermehrter Neudruck bildet einen Anhang zu:

(10) Simler, Josias. Vita Conradi Gesneri. 1566. (ZB: 5. 1655). - Die Epistola ad Turnerum ist nicht paginiert. Dagegen sind die aufgezählten und kurz kommentierten Werke G's mit Nr. 1-Nr. 72 bezeichnet.

Folgende dieser Werke sind linguistischen oder germanistischen Inhalts:

(11) Nr. 1: Lexicon Graocolatinum. 1537. (fehlt in ZB, vorhanden in der UB Basel: Ba I a 112). Ist in Basel bei Joh. Walder und nicht bei Henr. Petri erschienen, wie immer behauptet wird. 
(12) Nr. 21: Ambrosii Calepini dictionarium linguae Latinae. 1544. (Diese Ausgabe fehlt in ZB, hier vorhanden Ausgaben von 1551: ZB: 5.19 und 1558: ZB: Rq 304).

(13) Nr. 22: Onomasticon nominum propriorum. 1544. (ZB: AW 35).

(14) Nr. 51: Mithridates. 1555. (ZB: RR 1402).

(15) Nr. 56: Teutsche nammen der Fischen und Wasserthieren. 1556. (Sammelband De piscibus et aquatilibus libelli tres, p. $97 \mathrm{ff}$.). (ZB: XVIII 2031).

(16) Nr. 64: Maler, Josua. Dictionarium Germanico-Latinum: Einleitung. 1561. (ZB: 5. 98).

Ferner das von G. unter Nr. 23 erwähnte Vorwort:

(17) De utilitate ac dignitate linguae Graecae im Lexicon Graecolatinum. 1543 [nicht wie G. schreibt: 1544]. (ZB: 5. 96).

Am Schluß der Epistola ad Turnerum zählt G. seine damals in Vorbereitung befindlichen Werke auf.

Darunter für Sie von Interesse:

(18) Nomina propria Germanica. (verschollen).

(19) Origines Germanicorum vocabulorum. (verschollen).

In einem offenbar von Simler stammenden Corollarium ist unter den hinterlassenen Manuskripten G's unter anderen genannt:

(20) De scriptoribus Germanicis liber. (verschollen).

Ein Zusammenhang könnte bestehen zwischen (18) Nomina propria Germanica und:

(21) Handschriftliche Zusammenstellung altdeutscher Personennamen auf den hinteren Schmutzblättern von G's Handexemplar seiner Bibliotheca universalis (ZB: PA 18).

Alle nachfolgenden Hinweise habe ich nach den Kapiteln Ihrer geplanten Arbeit gegliedert :

zu Kap. 1

(22) Eine tabellarische Übersicht von G's Leben findet sich im Geßner-Gedenkband S. 21/22. (ZB: AX 1320).

(23) G's Entwicklung zum Sprachgelehrten und die Verflechtung seiner philologischen mit seinen übrigen Interessen ist ebenda S. 67/68 kurz gestreift.

zu Kap. 2

(24) G's sprachliche Auffassungen sind auch in Simlers Vita Conradi Gesneri mehrfach berührt. (ZB: $5.165_{5}$ ).

(25) Über die Bedeutung und Varianten verschiedener Personennamen äußert sich G. in seiner Bibliotheca universalis. 1545. (ZB: IV O 2).

(26) G's Absicht, den Mithridates neu herauszugeben, geht aus seinem Brief an Gasser vom 22. April 1563 hervor. (gedr. in Epist. med. libri III, 1577, fol. 28r: ZB: I B 66). 
zu Kap. 3

Im Mithridates zitiert G. als seine Quellen Werke folgender zeitgenössischer Autoren:

Aeneas Silvius (Piccolomini)

Agricola

Althamerus

Aventinus, Johannes (Turmair)

Balaeus, Johannes

Beatus Rhenanus

Bifrons, Jacobus (Bifrun)

Francus, Fabianus

Gelenius, Sigismundus

Georgewitz, Bartholomaeus
Glareanus, Henricus (Loris, Loriti)

Luther, Martin

Martyr, Petrus (Anglerius)

Miechow(ita), Matthias

Morus, Thomas

Münster, Sebastian

Postellus, Guilelmus

Stumphius, Johannes

Tschudi, Aegidius

Vadianus, Joachim (Watt)

Ich konnte bisher von folgenden Werken dieser Autoren Handexemplare Geßners feststellen:

(27) Bibliander, Theod. De restituenda pace in Germanico Imperio. s. a. (ZB: XXI 2354).

(28) Glareanus, Henr. De geographia. 1527. (ZB: XXVI $453 \mathrm{~b}_{1}$ ).

(29) Vadianus, Joach. De poetria. s.a. (ZB: Gal Tz 295).

Von diesen Handexemplaren kommt wohl nur das Werk des Glarean als Quelle für den Mithridates in Frage.

Kein Exemplar in G's Besitz hat sich in der ZB erhalten von:

(30) Bibliander, Theod. De ratione communi omnium linguarum. 1548. (ZB: Gal. 5.100). Geßner war dieses Werk sicher bekannt und es dürfte sich lohnen, dessen Einfluß auf den Mithridates zu untersuchen.

Weitere Hinweise auf im Mithridates zitierte zeitgen. Autoren:

(31) Aventinus: Über G's Absicht, die hss. Geschichtsbücher des Aventin zu erwerben, in welchen er gotische Lieder zu finden hoffte, vgl. Epist. med. libri III, fol. 25. (ZB: NNN 853).

(32) Bifrons: Vgl. das von G. herausgegebene Werk: Willich, Jodocus. Ars magirica ... Huic accedit Jacobi Bifrontis De operibus lactariis epistola. 1563. (ZB: XIV 2931).

(33) Tschudi: G. widmete ihm des Michael Ephesius Scholia in Aristotelis librum De longitudine ac brevitate vitae Aristotelis [in: Michaelis Ephesii scholia in Aristotelis libros. 1541. p. 41-76]. (ZB: St 394, ).

G's Handexemplare folgender Werke könnten für Sie noch von Nutzen sein:

(34) G's Bibliotheca universalis. 1545. (ZB: PA 18).

(35) Picardus, Joannes. De prisca celtopaedia. 1556. (ZB: 20. 124 2 ). Obschon ein Jahr nach dem Mithridates erschienen).

Als Nachweis der G. bekannten Literatur zu Ihrem Thema sind außer seiner Bibliotheca universalis von Bedeutung:

(36) G's Pandectarum libri XXI. 1548. (ZB: IV O 3). Besonders lib. I, der die Grammatik und Philologie behandelt.

(37) Appendix zur Bibliotheca universalis. 1555. (ZB: 5.13). 
Auch dienicht von G. besorgten Neuausgaben seinerBibliotheca universalis können weitere, G. nachträglich bekannt gewordene Literatur zum Thema nachweisen, besonders :

(38) Simler, Josias. Bibliotheca instituta a C. Gesnero in duplum aucta. 1574. (ZB: 5.14).

(39) Fries, J. J. Bibliotheca instituta a C. Gesnero amplificata. 1583. (ZB: 5.15).

G's Verhältnis zu den Buchdruckern ist untersucht worden von:

(40) Lutz, Hans. Konrad G's Beziehungen zu den Verlegern der Zeit nach seinen Pandekten. (Mélanges offerts à Marcel Godet. 1937). (ZB: CA 2029).

Über G's Zürcher Drucker orientieren:

(41) Staedtke, Joachim. Anfänge u. erste Blütezeit des Zürcher Buchdrucks. 1965. (ZB: BW 02.66).

(42) Leemann-van Elck, P. Die Offizin Froschauer. (Mitt. Antiquar. Ges. Zürich. Bd 33, Heft 2). 1940. (ZB: $00161 \mathrm{af}_{2}$ ).

(43) Leemann-van Elck, P. Die Offizin Geßner zu Zürich im 16. Jahrh. (Bibliothek der Schweizer Bibliophilen. Heft 15). 1940. (ZB: TA $320 \mathrm{~d}_{2}$ ).

Von G's Beziehungen zu den Buchdruckern ist auch einiges gesagt im

(44) Geßner-Gedenkband, S. 90-95. (in ZB: AX 1320).

Von auswärtigen Druckern kommen für den Philologen Geßner in Frage: in Basel Walder, Curio, Hospinian und Oporin; in Straßburg die beiden Richelius; in Paris Henricus Stephanus.

Bei den Fries'schen Wörterbüchern sind zu unterscheiden:

(45) Cholinus, Petrus et Jo. Frisius. Dictionarium Latinogermanicum. 1541. (ZB: 5.99). Dies ist der sog. «Kleine Fries».

(46) Frisius, Joannes. Dictionarium Latinogermanicum. Ed. 2 a. 1556. (ZB: 5.9). Dies ist der sog. «Große Fries».

G's nomenklatorische Neuschöpfungen auf botanischem, zoologischem und mineralogischem Gebiet können erst im «Großen Fries» berücksichtigt sein. Dagegen mag er für medizinische und philosophische Ausdrücke schon beim «Kleinen Fries» konsultiert worden sein.

Auf dem «Großen Fries» dürfte hauptsächlich beruhen:

(47) Maler, Josua. Die Teutsch spraach ... Dictionarium Germanicolatinum. 1561. (ZB: 5.98).

Über Malers Deutsch-lat. Wörterbuch finden sich einige Angaben im

(48) Geßner-Gedenkband S. 80-85. (in ZB: AX 1320).

zu Kap. $4 a$

G. hat mit Ausnahme von (55) kein deutsches Buch verfaßt, aber er bringt deutsches Sprachgut (Benennungen von Naturobjekten, Redewendungen, Sprichwörter usw.) in folgenden Werken:

(49) Catalogus alphabeticus animalium [in: Pandectarum libri XXI. 1548. fol. 221 r bis $233 \mathrm{v}$ ]. (ZB: $5.13_{2}$ ). 
(50) Historia quadrupedum viviparorum. 1551. (ZB: NNN 46).

(51) Historia quadrupedum oviparorum. 1554. (ZB: AW 28 $8_{1}$ ).

(52) Appendix historiae quadrupedum viviparorum et oviparorum. 1554. (ZB: 28 $8_{1}$ ).

(53) De avium natura. 1555. (ZB: AW 28 ${ }_{2}$ ).

(54) Icones avium omnium. 1555. (ZB: $\mathrm{AW} 1_{2}$ ).

(55) Teutsche nammen der Fischen und Wasserthieren. [in: De piscibus et aquatilibus omnibus libelli III novi. 1556. p. $97 \mathrm{ff}$.] (ZB: XVIII 2031).

(56) De piscium et aquatilium animantium natura. 1558. (ZB: NNN 48).

(57) Nomenclator aquatilium animantium. Icones animalium aquatilium. 1560. (ZB: NNN 44 $4_{3}$ ). Handexemplar G's.

(58) De serpentium natura. 1587. (ZB: NNN 431 ).

(59) Scorpionis insecti historia. 1587. (ZB: NNN 43.

(60) Moufetus, Thomas. Insectorum theatrum. 1634. (ZB: NNN 141). Verwertet u. a. G's entomologischen Nachlaß.

(61) Icones animalium quadrupedum viviparorum et oviparorum. 1553. (ZB: AW $1_{1}$ ).

(62) (Dasselbe). Ed. 2 a. 1560. (ZB: NNN 44, ). Handex. G's.

(63) Icones avium omnium. Ed. 2 a. (ZB: NNN 44, $)$. Handex. G's.

Über G's nomenklatorische Leistung in Bezug auf deutsche Vogelbenennungen vgl.:

(64) Geßner, Kd. Singvögel in C. Geßners Vogelbuch; bearb. v. Bernh. Milt. 1951. (ZB: DT 1018).

Auch in den von ihm verfaßten oder bloß druckfertig gemachten botanischen Werken ist G. bestrebt, neben den lateinischen Benennungen des Naturobjekts auch die deutschen mitzuteilen. Es kommen folgende Werke in Frage:

(65) Catalogus plantarum. 1542. (ZB: 5.183 $)$.

(66) Kyber, David. Lexicon rei herbariae. 1553. (ZB: NB 1501). (Darin von G. verfaßt: Tabulae collectionum in genere et particulatim per XII menses).

Die Tabulae collectionum sind, um ein alphabetisches Verzeichnis vermehrt, neu herausgekommen unter dem Titel:

(66 a) Geßner, Kd. De stirpium collectione tabulae. Hg. v. Caspar Wolf. 1587. (ZB: Z 2073).

(67) Cordus, Valerius. Annotationes in Dioscoridis de medica materia libros quinque. 1561. (ZB: I R 5). (Darin von G. verfaßt: De hortis Germaniae liber). Vgl. dazu:

(68) Fretz, Diethelm. Geßner als Gärtner. 1948. (ZB: CT 1055).

(69) De raris et admirandis herbis. 1555. (ZB: CT 1055). Bringt ebenfalls deutsche Pflanzennamen.

In den Legenden zu etwa 1000 für G's Historia plantarum bestimmten OriginalPflanzenzeichnungen, die sich heute in der Universitätsbibliothek Erlangen befinden, sind öfters auch deutsche Pflanzennamen vermerkt. Die ZB besitzt:

(70) Photokopien dieser Pflanzenzeichnungen unter den Signaturen Ms Z VIII 394 \& a-c, 395 .

Über die Geschichte und Wiederauffindung dieser Zeichnungen vgl.:

(71) Milt, Bernh. Conr. Geßner's Historia plantarum (Fragmenta relicta). (In: Vierteljahrsschrift Naturforsch. Ges. Zürich. 81, 1936, S. 285). (ZB: NUS 203 bq) 
Außer im

(72) Mithridates, 1555. (ZB: RR 1402), und im

(73) Vorwort zu Malers Deutsch-lat. Wörterbuch. 1561, (ZB: 5. 98), findet sich deutsches Sprachgut noch in G's Schriften:

(74) Libellus de lacte. 1541. (ZB: VI 330).

(75) Descriptio Montis Fracti. (In: De raris et admirandis herbis. 1555). (ZB: 5. 200 ${ }_{1}$ ).

(75 a) De anima liber 1563. (ZB: VII 381).

(76) De rerum fossilium lapidum et gemmarum maxime, figuris et similitudinibus. 1565. (ZB: 5. 312 $)$.

Ferner sporadisch in der publizierten Korrespondenz G's:

(77) Epistolarum medicinalium libri III. 1577. (ZB: NNN 853).

(78) Epistolarum medicinalium liber IV. 1584. (In der ZB vorhanden bloß im Nachdruck bei Hanhart, Joh. Conrad Geßner. 1824. S. 301-345 (ZB: IV 0 486).

Diese vier Briefbände liegen zur Hauptsache ins Deutsche übersetzt vor in:

(79) Peine, Hans. Briefe Kd. Geßners an seine Freunde Gasser und Culmann. Diss. med. Düsseldorf. 1939. (ZB: DP 1421).

(80) Helmich, Egon. Geßners Briefe an Crato v. Krafftheim. Diss. med. Düsseldorf. 1938. (ZB: DP 1420).

(81) Peine, Joseph. Das dritte Buch der medizinischen Briefe von Kd. Geßner. Diss. med. Düsseldorf. 1941. (ZB: Phot 325).

G's Briefe an Joh. Bauhin sind gedruckt in:

(82) Bauhinus, Joannes. De plantis a divis sanctisve nomen habentibus. Additae sunt Conr. Gesneri epistolae. 1591. (ZB: 249. ). $^{2}$

Nur handschriftlich erhaltene Briefe, die für Sie von Interesse wären, sind in der ZB nicht vorhanden, mit Ausnahme von zwei unter (125) und (126) erwähnten Briefentwürfen.

$z u$ Kap. 4 b

Dafür, daß G. sich zur Beschaffung seines sprachlichen Materials schon der Fragebogenmethode bedient hätte, scheinen mir keine Anhaltspunkte vorzuliegen. Meines Wissens war der früheste, von einem Schweizer Forscher in Umlauf gesetzte Fragebogen die

(83) Charta invitatoria, quaestionibus, quae historiam helvetiae naturalem concernunt, praefixa, 1699, verfaßt von Johann Jakob Scheuchzer (1672-1733), der allerdings in Geßner sein Vorbild gesehen und diesen in mancher Hinsicht nachgeahmt hat.

Vgl. dazu:

(84) Furrer, Ernst. Polyhistorie im alten Zürich vom 12. bis 18. Jahrhundert. (Vierteljahrsschrift der Naturforschenden Gesellschaft Zürich. 110, S. 363-394, speziell S. 384). (ZB: NUS 203, 110). 
G. hat sich sein sprachliches und sein naturwissenschaftliches Material (beides ging bei ihm Hand in Hand) wohl hauptsächlich auf folgende Weise beschafft:
A) durch Lektüre
B) durch Unterhaltung mit Gelehrten
C) auf dem Korrespondenzwege
D) durch Befragung von Berufsleuten
E) durch Ausfragung von fremden Gästen
F) mittelst Suchlisten
G) mittelst Austauschlisten
H) durch Vermittlung seiner Werke.

zu $A$

Außer den zwischen (26)/(27) aufgezählten 20 zeitgenössischen Autoren zitiert G. im Mithridates 20 antike und 3 mittelalterliche. Es sind dies:

$\begin{array}{ll}\text { Appianus Alexandrinus } & \text { Homer } \\ \text { Aristoteles } & \text { Johannes Grammaticus } \\ \text { Berosus } & \text { Livius } \\ \text { Cicero } & \text { Notker } \\ \text { Clemens Alexandrinus } & \text { Porphyrius } \\ \text { Diodor } & \text { Sallust } \\ \text { Ennius } & \text { Saxo Grammaticus } \\ \text { Euphorius } & \text { Strabo } \\ \text { Evangelium Matthaei } & \text { Tacitus } \\ \text { Festus } & \text { Varro } \\ \text { Herodot } & \text { Xenophon } \\ \text { Hippocrates } & \end{array}$

Von Werken dieser Autoren konnten bisher in der ZB folgende als Handexemplare G's aufgefunden werden:

(85) Aristoteles. Opera omnia. Per Desid. Erasmum. Basileae 1539. (ZB: B 83 \& a).

(86) Aristoteles. Opera omnia. Basileae 1542. (ZB: XXIII 41 \& 42).

(87) Novum Testamentum iam quintum recogn. à Des. Erasmo Roterod. Basileae, Froben 1535. (ZB: Gal Tz 12).

(88) Hippocrates. Opera omnia. Per Janum Cornarium in lingua Latina conscripta. Basileae, Frobenius 1546. (ZB: Md A 21).

(89) Homerus. Ilias et Ulyssea cum interpret. Basileae, Hervagius 1535. (ZB: Gal II App. $63_{1,2}$ ).

(90) Johannes Grammaticus. Libri 25 de mundi aeternitate. Venetiis, Hier. Scotus 1551. (ZB: $\left.3.16_{4}\right)$.

(91) Porphyrius Philosophus. De non nocandis ad epulandum animantibus libri IIII. Eiusdem Selectae breves sententiae. Florentiae, Bern. Juncta 1548. (ZB: 3. 16 ${ }_{2}$ ).

(92) Tacitus. Annalium libri sedecim. Basileae, Frobenius 1533. (ZB: Dr. Ms 430 ${ }_{2}$ ). 
(93) Xenophon. Opera. Basileae, Cratander 1533. (ZB: Dr. Ms 430 $)$.

Die genaueren Titel der Werke, aus denen G. geschöpft hat und die im Mithridates z.T. gekürzt angeführt sind, können ersehen werden aus G's bibliographischen Werken [aufgezählt oben unter (34), (36)-(39)].

$z u B$

Im Hause des J. J. Ammann war G. der Tischgenosse Biblianders gewesen. Die Ideen dieses Gelehrten, der später (1548) seine Ansicht vom gemeinsamen Ursprung aller Sprachen verteidigte, vgl. (30) haben G. sicher beeinflußt, was im Nachwort zum Mithridates angetönt wird. Auch in Gesprächen mit seinen Lehrern Pellikan (Zürich), Wolmar (Bourges) und mit seinen Freunden Joh. Fries (Zürich), Achilles Gasser und Georg Laetus (beide in Augsburg) und Arnoldus Peraxylus Arlenius (Venedig) dürfte das Wunder der Sprache öfters Gegenstand der Unterhaltung gewesen sein. $\mathrm{Da} ß$ er sich auch mit seinen Schülern über linguistische Probleme unterhalten hat, bezeugt:

(94) Simler, Josias. Vita Conradi Gesneri. 1566. fol. 7r. (ZB: 5. 165 5 ). Vgl. auch:

(95) Geßner-Gedenkband S. 80. (ZB: AX 1320).

$z u C$

Listen der Leute, die G. für seine Tierbücher Material lieferten und außer einigen in Zürich seßhaften wohl alle Korrespondenten G's waren und ihm auch Tiernamen in verschiedenen Sprachen mitteilten, finden sich am Anfang folgender Werke:

(96) Historia quadrupedum viviparorum. 1551. (ZB: NNN 46). 52 Namen.

(97) De piscium et aquatilium animantium natura. 1558. (ZB: NNN 48). 27 Namen.

Die botanischen Korrespondenten G's sind zusammengestellt in:

(98) Geßner, Kd. Conradi Gesneri opera botanica; ed. Cas. Christ. Schmiedel. I, 1753, p. XLII. (ZB: NB 9). 61 Namen.

Den unter (77)-(82) erwähnten gedruckten Briefsammlungen G's ist noch beizufügen:

(99) Rath, Gernot. Die Briefe Konrad Geßners aus der Trew'schen Sammlung. (Gesnerus 7, 1950, S. 140-170; 8, 1951, S. 195-215). (In ZB: XE 506, 1950, 1951).

Viele weitere Briefe G's aus der Vadiana (St. Gallen), dem Staatsarchiv Zürich und der Zentralbibliothek Zürich finden sich übersetzt, resumiert oder ausgewertet bei:

(100) Hanhart, Joh. Conrad Geßner. 1824. (ZB: IV O 486).

Alle diese Briefsammlungen zusammen mit einigen weiteren einzeln publizierten Briefen und den bisher bekannt gewordenen nur handschriftlich vorhandenen, bilden nur einen kleinen Bruchteil der Korrespondenz Geßners. Der Rest ist verschollen. Typisch für die Art, wie G. sich das sprachliche Material auf dem Korrespondenzwege beschaffte, ist: 
(101) G's Erkundigung nach den im Rhein bei Basel vorkommenden Fischen und deren Benennungen in seinem Brief an Joh. Hospinian vom 8. Okt. 1557. (Epist. med. libri III, 1577, fol. 102 r). (ZB: NNN 853).

$z u D$

Daß G. den Berufsleuten (Jägern, Fischern, Vogelstellern, Hirten etc.) viele Auskünfte verdankte, ergibt sich aus der

(102) Vorrede an den Leser in der Historia animalium lib. I, 1551. (ZB: NNN 46).

Von der Art solcher Auskünfte gibt ein gutes Bild der Bericht von

(103) Heinrich Dettling von Schwyz über die Käsebereitung, datiert vom 28. Sept. 1552. (ZB: Ms C. 50a Nr. 1 und 68).

$z u E$

Über G's Gastfreundschaft äußert sich

(104) Simler, Josias. Vita Conradi Gesneri. 1566. fol. 17r. (ZB: 5.1655). Stelle übersetzt im Geßner-Gedenkband S. 231. (ZB: AX 1320).

In einem noch erhaltenen, in die USA verkauften Gästebuch G's mit Einträgen aus den Jahren 1555-1565 haben sich 227 Besucher eingetragen. Die ZB besitzt eine

(105) Photokopie von Geßners Gästebuch. (Ms Z VIII 759). Über das letztere vgl.:

(106) Durling, Richard. Conrad Gesner's Liber amicorum. (Gesnerus 22, 1965, S. 134-159), (ZB: XE 506, 1965), und:

(107) Geßner-Gedenkband S. 228-230. (ZB: AX 1320).

$z u F$

Daß G. an naturhistorisch interessierte Freunde Suchlisten geschickt hat um in den Besitz von ihm noch fehlenden Naturgegenständen, Abbildungen oder Benennungen von solchen zu kommen, ist wahrscheinlich, kann aber vorläufig nicht bewiesen werden. Dagegen hat er eine solche Suchliste, aus welcher ersichtlich war, welche Tierabbildungen ihm noch fehlten, eingerückt in:

(108) Pandectarum libri XXI. 1548. fol. 221 r ff. (ZB: 5. 13 $3_{2}$ ).

$z u G$

G. hat mit den Botanikern unter seinen Freunden einen regen Austausch von frischen und getrockneten Pflanzen, Samen und Wurzeln, sowie von Pflanzenabbildungen gepflegt und mit ihnen u.a. auch über die Benennungen der betr. Pflanzen diskutiert, wobei ihn namentlich auch die deutschen Namen interessierten.

Dies geht namentlich aus G's Korrespondenz mit Aretius, Joh. Fabritius, Fuchs, Guilandinus, Obrecht, Occo, Felix Platter und Zwinger hervor, deren erhaltene Reste publiziert sind in: 
(109) Epist. med. libri III. 1577. (ZB: NNN 853).

Ferner aus der Korrespondenz mit Joh. Bauhin in:

(110) Bauhinus, Joannes. De plantis a divis sanctisve nomen habentibus. Additae sunt Conr. Gesneri epistolae. 1591. (ZB: 2491).

G's Arbeit De hortis Germaniae war zur Hauptsache als Austauschliste gedacht. Sie ist publiziert als Zugabe zu seiner Ausgabe der Werke des Valerius Cordus:

(111) Cordus, Valerius. Annotationes in Dioscoridis de medica materia libros quinque. 1561. (ZB: I R 5).

zu $\mathrm{H}$

G. hat nach Möglichkeit seine eigenen Werke in den Dienst weiterer Materialbeschaffung gestellt. Selten versäumt er in den Widmungen seiner Werke und in den Vorreden an den Leser die Bitte anzubringen, man möchte sein Vorhaben durch Mitteilungen, Berichtigungen, Zusendung von Naturalien und Abbildungen unterstützen, da er als wenig vermöglicher Gelehrter auf die kostenlose Mithilfe vieler angewiesen sei. Die Widmung jedes Buches seiner Pandekten an einen berühmten Verleger und die Beifügung von Verlagslisten erfolgte wahrscheinlich in der Absicht, diese Verleger zu Bücherschenkungen an G. zu bewegen. Bücher waren die Hauptquellen, aus denen er für seine Arbeiten schöpfte; aber seine beschränkten Mittel erlaubten viel zu wenig Anschaffungen. Manche Verleger haben G. in der Tat mit Schenkungen ihrer Verlagsprodukte ausgeholfen. 8 solcher Verlegergeschenke, die aber Ihr Thema nicht berühren, haben sich in der ZB erhalten:

(112) Geschenke der Verleger Constantin, Paris (ZB: 20. 951), Episcopius, Basel (ZB: V A 8), (ZB: 20.954), Froben, Basel (ZB: V A 8), (ZB: 20.601 ), Oporin, Basel (ZB: XVIII 280 ${ }_{10}$ ), Stephanus, Henr., Paris (ZB: $3.16_{1}$ ), Stephanus, Rob., Paris (ZB: $\left.3.16_{1}\right)$, (ZB: St $\left.37_{1}\right)$.

Von nicht zu unterschätzendem Einfluß auf die Materialbeschaffung ist gewesen, daß G. als erster Zoologe jede seiner Tierbeschreibungen mit Abbildungen und mit den Namen des Tieres in verschiedenen Sprachen versehen hat. Dadurch forderte er in weit stärkerem Maße als mit einer bloß verbalen Darstellung die Zustimmung oder Kritik der Leser und damit zugleich deren Helferwillen heraus. In der gleichen Absicht zögerte G. auch nicht, in Ermangelung von guten Abbildungen auch schlechte, von ihm nicht gebilligte, zu bringen. Die Reaktion darauf war gewöhnlich die, daß sich irgend ein Leser entschloß, ihm eine bessere Abbildung zu schicken. Hand in Hand mit solchen Berichtigungen gingen auch solche der Nomenklatur und ergänzende Angaben.

Auf alle unter A-H geschilderten Wegen und Umwegen hat G. sein Material gesammelt. Die Eruierung der Tier- und Pflanzennamen und ihrer Synonyma in den verschiedenen Sprachen hat ihm, wie er selbst gesteht, oft mehr Mühe gekostet als alles übrige. Sein primäres Interesse an der Nomenklatur der Naturobjekte war wohl kaum ein sprachliches, sondern ein naturwissenschaftliches. Es bestand in der Notwendigkeit, die Synonyma zu kennen, um das Wissen über den Gegenstand aus möglichst vielen Sprachgebieten und Sprachepochen sammeln zu können. Aber es 
ist natürlich, daß er als geschulter und geborener Philologe auch ein besonderes Gefühl für die linguistische Aussage der Nomenklatur mitbrachte, und er wird daraus, vielleicht nicht ohne Anregung von außen (Bibliander), die Schlüsse gezogen haben, die sich zusammen mit andern Beobachtungen zur Idee von der Verwandtschaft gewisser Sprachen verdichtet haben.

Vielleicht lassen sich aus den handschriftlichen Notizen, die G. in seinen Handexemplaren eigener Werke angebracht hat, weitere Einblicke in die Art und Weise gewinnen, wie er sein Material gesammelt hat. Von solchen Handexemplaren haben sich in der ZB erhalten:

(113) Historia plantarum. 1541. (ZB: D 459a).

(114) Icones animalium quadrupedum. Ed. 2 a. 1560. (ZB: NNN 44 $4_{1}$ ).

(115) Icones avium. Ed. 2 a. (ZB: NNN 44, $4_{2}$.

(116) Icones animalium aquatilium. 1560. (ZB: NNN 44, $4_{3}$.

(117) Bibliotheca universalis. 1545. (PA 18).

zu Kap. 4 c

Von seinen Übersetzungen in moderne Sprachen stammt keine von G. selbst; dieser hat nur lateinisch und griechisch publiziert.

Zu G's Lebzeiten sind von seinen Werken ins Deutsche übersetzt worden:

(118) Köstlicher Schatz Evonymi Philiatri. [Teil I]. 1555. Übersetzer: Joh. Rud. Landenberger. (ZB: AW 30071).

(119) Vogelbuch. 1557. Übersetzer: Rudolf Hüsli. (ZB: NS $44_{1}$ ).

(120) Thierbuch. 1563. Übersetzer: Johann Herold (bis Blatt LV), Konrad Forer (Rest). (ZB: NS $4{ }_{2}$ ).

(121) Fischbuch. 1563. Übersetzer: Konrad Forer. (ZB: NS $44_{3}$ ).

Anhaltspunkte für eine mögliche Beratung der Übersetzer durch den Autor dürften sich aus den Vorreden ergeben. Es will mir allerdings scheinen, daß die Übersetzungen mehr ein kommerzielles Unternehmen des Verlegers Froschauer als ein wissenschaftliches Anliegen G's darstellen.

$z u$ Kap. $4 d$

Zusammenhängende deutsche Texte G's gibt es nur wenige. Mit Ausnahme von (55) sind sie nur handschriftlich vorhanden, so daß zusammen mit den unter (49)-(82) aufgezählten Werken mit ihren gelegentlichen deutschen Einschiebseln nur wenig Material existiert um G's Sprache zu beurteilen.

Die in der ZB vorhandenen handschriftlichen Deutschtexte G's sind:

(122) Ein consilium zur bewarung der gesunttheit ... Ohne Datum. (ZB: Ms C 50a Nr. 63 = Blatt 320-327). Autograph.

(123) Entwurf einer Eingabe G's an den Rat der Stadt Zürich betreffend die Einrichtung eines botanischen Gartens. Ohne Datum. Nur in einer Abschrift des 18. Jahrhunderts erhalten (ZB: Ms S 93, Nr. 181). Abgedruckt bei:

(124) Fretz, Diethelm. Konrad Geßner als Gärtner. 1948. S. 94/95. (ZB: CT 3055). 
(125) Ein ausführliches und ein gekürztes handschriftliches Widmungsschreiben an den Bürgermeister und Rat der Stadt Zürich. Einem diesen überreichten Exemplar von G's Historia animalium lib. I, 1545, vorgebunden. (ZB: 5.1 a). Autograph ist nur G's Unterschrift.

(126) Autographer Entwurf von G's Gesuch an den Bürgermeister und Rat der Stadt Zürich um Dotierung mit einer Chorherrenpfründe. 1558. (ZB: Ms C 50 a Nr. 33 $=$ fol. 225r-226r). Teilfaksimile und Abdruck des ganzen Briefes in:

(127) Geßner-Gedenkband S. 224-227. (ZB: AX 1320).

(128) Consilium für epileptischen Knaben. Ohne Datum. (ZB: Ms S 204a, 1 Seite). Autograph. Faksimile und Transkription in:

(129) Geßner-Gedenkband S. 118/119. (ZB: AX 1320).

(130) Consilium für epileptisches Mädchen. Ohne Datum. (ZB: Ms S 204a, 21/2 Seiten). Autograph.

(131) Consilium für Gelbsuchtpatienten Balthasar Kuchemeister. Ohne Datum. (ZB: Ms S 204b, fol. 225r). Autograph. Stark reduziertes Faksimile eines Teiles davon in:

(132) Fischer, Hans. Conrad Geßner. (Neujahrsbl. Natf. Ges. Zürich. 168, 1966), S. 83. (ZB: KK 562, 168).

(133) Autographer Entwurf von G's Testament betr. seinen Wappenbrief und eine Familienstiftung. Datiert 18.Sept. 1564. (ZB: F-A Geßner $1516_{3}$, Nr. 2). 6 Seiten. Autograph.

Vielleicht die einzigen längeren wissenschaftlichen Texte G's in deutscher Sprache sind zwei autographe Pflanzenbeschreibungen:

(134) Laserpitium Siler L. (ZB: Ms Z VIII 394a, Blatt 64a) und

(135) Vaccinium uliginosum L. (ZB: Ms Z VIII 395, Blatt 383).

21.6.1967

Zentralbibliothek Zürich

R. Steiger 